\title{
1 Information flows from hippocampus to cortex during replay of verbal working
} 2 memory items.

3

4 Vasileios Dimakopoulos ${ }^{1}$, Lennart Stieglitz ${ }^{1}$, Pierre Mégevand ${ }^{2,3}$, Johannes

5 Sarnthein ${ }^{1,4^{*}}$

6

71 Klinik für Neurochirurgie, Universitätsspital Zürich, Universität Zürich, 8091 Zurich,

8 Switzerland

92 Département des neurosciences fondamentales, Faculté de médecine, Université

10 de Genève, Geneva, Switzerland

113 Service de neurologie, Hôpitaux Universitaires de Genève, Geneva, Switzerland

124 Neuroscience Center Zurich, ETH Zurich, 8092 Zurich, Switzerland

13

14 vasileios.dimakopoulos@usz.ch

15 lennart.stieglitz@usz.ch

16 pierre.megevand@unige.ch

17 johannes.sarnthein@usz.ch

$18{ }^{*}$ Correspondence should be addressed to J.S.

19

20 Keywords: Granger causality, phase-locking value, auditory cortex, posterior cortex,

21 beta rhythm 


\section{ABSTRACT}

23 Background: The maintenance of items in working memory (WM) relies on a

24 widespread network of cortical areas and hippocampus where synchronization

25 between electrophysiological recordings reflects functional coupling. We investigated

26 the direction of information between sensory areas and hippocampus during

27 encoding and maintenance of WM items.

28 Methods: Participants $(\mathrm{N}=15)$ performed a WM task where a string of letters was

29 presented all at once, thus separating the encoding period from the maintenance

30 period. Participants mentally replayed the letters during maintenance. We recorded

31 sEEG from the hippocampus, scalp EEG and, additionally in 3 participants, temporo-

32 parietal ECoG.

33 Results: When analyzing the information flow to and from auditory cortex by

34 Granger causality, the flow was from ECoG over auditory cortex to hippocampus

35 with a peak in the $12-24 \mathrm{~Hz}$ beta range while letters were presented, and this flow

36 was subsequently reversed during maintenance, while letters were maintained in

37 memory. The same pattern appeared to and from hippocampus with ECoG over

38 temporo-parietal cortex. For scalp EEG, the pattern appeared on temporal sites,

39 albeit in the $4-12 \mathrm{~Hz}$ theta-alpha range. While the pattern was significantly structured

40 for correct trials, it was unstructured for incorrect trials.

41 Conclusions: The functional interaction between hippocampus and cortex and

42 the reversal of information flow provide a physiological basis for the encoding of

43 memory items and their active replay during maintenance. 


\section{$45 \quad$ SIGNIFICANCE STATEMENT}

46 How do we encode and recall memories to guide action within seconds? Here, we

47 investigated electrical activity in hippocampus and cortex while the participants

48 mentally replayed a set of letters to maintain them working memory with the aim to

49 respond correctly. We found clear evidence that during the encoding of the letters,

50 the information flow was from sensory cortex to hippocampus (bottom-up). The flow

51 was subsequently reversed during maintenance (top-down), thus providing a

52 physiological basis for the recall of the memory items. This functional interaction

53 provides the first evidence of bidirectional communication during encoding and the

54 active replay of memory items.

\section{INTRODUCTION}

56 Working memory (WM) describes our capacity to represent sensory input for

57 prospective use $(1,2)$. Maintaining content in WM requires collaboration within a

58 widespread network of brain regions. The anatomical basis of WM was shown

59 noninvasively with EEG / MEG (3-9) and invasively with intracranial EEG (10-19) and

60 single unit recordings (19-22).

61 In cortical brain regions, WM maintenance correlates with sustained neuronal

62 oscillations, most frequently reported in the theta-alpha range $(4-12 \mathrm{~Hz})(3,5-7,10$,

$6311,13,19,23)$. Also in the hippocampus, WM maintenance was associated with

64 sustained theta-alpha oscillations $(14,19)$. As a hallmark for WM maintenance,

65 persistent neuronal firing was reported during the absence of sensory input,

66 indicating the involvement of the medial temporal lobe in WM (19-21).

67 At the network level, synchronized oscillations have been proposed as a mechanism for functional interactions between brain regions $(24,25)$. It is thought

69 that these oscillations show temporal coupling of the low-frequency phase for long-

70 range communication between cortical areas $(4,6,13,17-19,26)$. This

71 synchronization suggests an active maintenance process through reverberating 72 signals between brain regions.

73 We here extend previous studies with the same task (3), which had shown parietal 74 activation and strong scalp EEG synchronization between stereotactic EEG (sEEG)

75 in the hippocampus and scalp EEG (19). In addition to scalp EEG and hippocampal

76 sEEG, three participants of this study had cortical recordings (ECoG) from 
77 electrodes over primary auditory, parietal and occipital cortical areas. Given the low

78 incidence of the epileptogenic zone in parietal cortex, parietal ECoG recordings are

79 rare. We found that the information flow was from sensory areas to hippocampus

80 during the encoding of WM items and the flow was from hippocampus to cortex for

81 the replay of the items during the maintenance period.

\section{$82 \quad$ RESULTS}

\section{$83 \quad$ Task and behavior}

84 Fifteen participants (median age $29 \mathrm{y}$, range [18-56], 7 male) performed a 85 modified Sternberg WM task (71 sessions in total, 50 trials each). In the task, items 86 were presented all at once rather than sequentially, thus separating the encoding 87 period from the maintenance period. In each trial, the participant was instructed to 88 memorize a set of 4, 6 or 8 letters presented for $2 \mathrm{~s}$ (encoding). The number of 89 letters was thus specific for the memory workload. The participants read the letters 90 themselves and heard them spoken at the same time. After a delay (maintenance) 91 period of $3 \mathrm{~s}$, a probe letter prompted the participant to retrieve their memory 92 (retrieval) and to indicate by button press ("IN" or "OUT") whether or not the probe 93 letter was a member of the letter set held in memory (Fig. 1a). During the 94 maintenance period, participants rehearsed the verbal representation of the letter 95 strings subvocally, i.e. mentally replayed the memory items. This activation of the 96 phonological loop (1) is a component of verbal WM as it serves to produce an 97 appropriate behavioral response (2).

98 The mean correct response rate was $91 \%$ (both for IN and OUT trials). The rate of correct responses decreased with set size from a set size of 4 (97\% correct

100 responses) to set sizes of $6(89 \%)$ and 8 (83\%) (Fig. 1 b). Across the participants, 101 the memory capacity averaged 6.1 (Cowan's K, (correct IN rate + correct OUT rate $1021)^{*}$ set size), which indicates that the participants were able to maintain at least 6 103 letters in memory. The mean response time (RT) for correct trials (3045 trials) was

$1041.1 \pm 0.5$ seconds and increased with workload from set size $4(1.1 \pm 0.5 \mathrm{~s})$ to 6 (1.2 $105 \pm 0.5 \mathrm{~s})$ and $8(1.3 \pm 0.6 \mathrm{~s}), 53 \mathrm{~ms} /$ item (Fig. $1 \mathrm{c})$. Correct IN/OUT decisions were 106 made more rapidly than incorrect decisions ( $1.1 \pm 0.5$ versus $1.3 \pm 0.6$ seconds).

107 These data show that the participants performed well in the task and that the 108 difficulty of the trials increased with the number of letters in the set. In further 
109

analysis, we focused on correct trials with set size 6 and 8 letters to assure hippocampal activation and hippocampo-cortical interaction as shown earlier (19).

\section{Power spectral density in cortical and hippocampal recordings}

To investigate how cortical and hippocampal activity subserves WM processing, we analyzed sEEG recorded in the hippocampus (Fig. 1 d) together with ECoG from cortical strip electrodes (Fig. 2 a, Fig. 3 a, f). In the following, we present power spectral density (PSD) time-frequency maps from representative electrode contacts.

In an occipital recording of Participant 1 (grid contact H3, Fig. 2 a), strong gamma activity (> $40 \mathrm{~Hz}$ ) in the relative power spectral density (PSD) occurred while the participant viewed the letters during encoding (Fig. 2 b). After the letters disappeared from the screen, activity occurred in the beta range $(12-24 \mathrm{~Hz})$ towards the end of the maintenance period. In a temporal recording (grid contact C2) gamma activity occurred during encoding, thus confirming the anatomical localization of this contact over auditory cortex (supplementary Fig. S1 a). Similarly, the temporal scalp EEG of Participant 2 (electrode site T3, Fig 3 a) showed activity during encoding and maintenance, albeit at lower frequencies (Fig 3 b). In Participant 3, a similar pattern occurred in the PSD of a temporo-parietal recording (most anterior strip electrode contact, Fig $\mathbf{3} \mathbf{f}$ ), where the appearance of the probe letter again prompted gamma activity. This site coincides with the generator of scalp EEG that was found in the parietal cortex for the same task (3). The PSD thereby confirmed the findings of local synchronization of cortical activity during WM maintenance $(3,8,9)$.

In the hippocampus of all three participants, we found elevated activity in the beta range (12-24 Hz) towards the end of the maintenance period (Fig 2 c, Fig $3 \mathbf{~ c , h}$ ), confirming the hippocampal contribution to processing of this task (19)

\section{Directed functional coupling between hippocampus and ECoG}

To investigate the functional coupling between cortex and hippocampus, we first calculated the phase locking value (PLV). During maintenance, we found enhanced PLV in the beta range (supplementary Fig. S1 b). The highest PLV occurred from the contact C2 over auditory cortex (Fig 2 d), which speaks for a functional coupling between auditory cortex and hippocampus mediated by synchronized oscillations (24).

What was the directionality of the information flow during encoding and maintenance in a trial? We used spectral Granger causality (GC) as a measure of 
142 directed functional connectivity to determine the direction of the information flow

143 between auditory cortex and hippocampus during the trials. During encoding, the

144 information flow was from auditory cortex to hippocampus with a maximum in the

145 beta frequency range (12-24 Hz, dark blue curve in Fig. 2 e). During maintenance,

146 the information flow in the same frequency range was reversed (dark red curve), i.e.

147 from hippocampus to auditory cortex. Concerning the spatial distribution of the beta

$148 \mathrm{GC}$, the highest GC during encoding occurred from auditory cortex to hippocampus

149 (supplementary Fig. S1 c). During maintenance, the beta GC was high from

150 hippocampus to both auditory cortex and parietal cortex (supplementary Fig. S1 d).

151 As a further illustration of the GC time-course, the time-frequency plot shows the

152 difference between GC spectra ( $\Delta$ Granger) at each time point, where blue indicates

153 net flow from auditory cortex to hippocampus and red indicates net flow from

154 hippocampus to auditory cortex Fig. 2 f).

155 Similarly in Participant 2, the time course of GC followed the same pattern

156 between auditory cortex (anterior strip electrode contact in Fig. 3 a) and

157 hippocampus (Fig. 3 d,e). Among all participants that had both sEEG and temporo-

158 parietal ECoG recordings, Participant 3 had an electrode contact over visual cortex;

159 the sensory localization was indexed by the strong gamma activity (most posterior

160 contact of the strip electrode in Fig. $3 \mathbf{f}$ ). The time-course of information flow

161 between visual cortex and hippocampus (Fig. $3 \mathbf{i , j}$ ) followed the same pattern as

162 described for the auditory cortex above. Thus, letters were encoded with information

163 flow from sensory cortex to hippocampus; conversely, the information flow from

164 hippocampus to sensory cortex indicated the replay of letters during maintenance.

165 Directed functional coupling between hippocampus and scalp EEG

166 We then confirmed the directed functional coupling in the whole group of $\mathrm{N}=15$

167 participants. We calculated GC between all hippocampal channels and all scalp EEG

168 electrodes. We present here the electrode pairs with the highest $\Delta$ Granger values. It

169 turned out that in each participant, the highest GC occurred in temporal electrodes

170 over auditory cortex; in participants where temporal sites were not recorded from, the

171 highest GC occurred at the neighboring electrode sites C3 or C4.

172 The GC time-course in Participant 1 was similar for scalp EEG (Fig. 4 a) and

173 ECoG (Fig. 2 e). We found high GC between temporal EEG over auditory cortex

174 (EEG site T4) and hippocampus. The GC was lower than for ECoG, as expected for 
175 the lower signal amplitude of scalp EEG. During encoding, the information flow was

176 from temporal EEG to hippocampus in the alpha frequency range (dark blue curve).

177 During maintenance, the information flow range was reversed (dark red curve), i.e.

178 from hippocampus to auditory cortex. In Participant 2, the information flow was high

179 from electrode T5 to hippocampus during encoding in the alpha range (dark green

180 line in Fig. 4 b). In Participant 3, the information flow was highest from hippocampus

181 to electrode T6 during maintenance in the theta range (dark red line in Fig. 4 c). Also

182 for all other participants (Fig. 4 d-o), all GC spectra during encoding had frequency

183 ranges where information flow from cortex to hippocampus (encoding, dark blue

184 curve) was significantly higher than in the reverse direction (light blue curve) and

185 where information flow from hippocampus to cortex (maintenance, dark red curve)

186 was significantly higher than in the reverse direction (light red curve). We then

187 calculated the time-frequency plots of the $\Delta$ Granger for each participant; the

188 averaged plot illustrates the predominant flow from auditory cortex to hippocampus

189 during encoding (blue) and flow from hippocampus to auditory cortex (red) during

190 maintenance in the theta-alpha frequency range (4-15 Hz, Fig. 5a).

191 Finally, we looked at the effect of behavior on $\Delta$ Granger. For each participant we

192 calculated the $\Delta$ Granger for encoding and maintenance in the respective frequency

193 bands of significance. For correct trials, the $\Delta$ Granger was negative during encoding

194 and positive during maintenance for all 15 participants $(P=0.005$, paired cluster

195 based permutation test, Fig. 5 b). For incorrect trials, the same analysis was not

196 significant $(P=0.0647$, paired cluster based permutation test, Fig. 5 c) for incorrect

197 trials. This suggests that timely information flow, as indexed by GC, is relevant for

198 producing a correct response.

\section{DISCUSSION}

200 Working memory (WM) describes our capacity to represent sensory input for

201 prospective use. Our findings suggest that this cognitive function is subserved by

202 bidirectional oscillatory interactions between the hippocampus and the sensory-

203 parietal cortex as indicated by phase synchrony and Granger causality. In our verbal

204 working memory task, the encoding of letter items is isolated from the maintenance

205 period in which the active rehearsal of memory items is central to achieve correct

206 performance. First, analysis of task-induced power showed sustained oscillatory 
207 activity in cortical and hippocampal sites during the maintenance period. Second, 208 analysis of the inter-electrode phase synchrony and the directional information flow 209 showed task-induced interactions between cortical and hippocampal sites. The 210 oscillations were in the beta band when recorded with intracranial electrodes and, 211 when recorded with scalp electrodes, extended down to the theta-alpha band. Third, 212 the directional information flow was from sensory cortex to hippocampus during 213 encoding and, during maintenance, the reverse flow occurred from hippocampus to 214 cortex. Fourth, the comparison between correct and incorrect trials suggests that the 215 participants relied on timely information flow to produce a correct response. Our data 216 suggests a surprisingly simple model of information flow within a network that 217 involves sensory cortices and hippocampus (Fig. 5 d): During encoding, letter strings 218 are verbalized as melody. The incoming information flows from sensory cortex to 219 hippocampus (bottom-up). During maintenance, participants actively recall and 220 rehearse the melody. The Granger causality indicates the information flow from 221 hippocampus to cortex (top-town) as the physiological basis for the replay of the 222 memory items, which finally guides action.

223 The current study is embedded in previous studies using the same or similar 224 tasks. Persistent firing of hippocampal neurons indicated hippocampal involvement 225 in the maintenance of memory items (19-21). Parietal generators of theta-alpha EEG 226 indicated involvement of parietal cortex in WM maintenance $(3,5,7,19,27)$. The 227 hippocampo-cortical phase synchrony (PLV) was high during maintenance of the 228 high workload trials (19). Building on these previous studies, the current study 229 focused on high workload trials and extended them by the analysis of directional 230 information flow.

231 In the literature, there are several studies investigating the WM network. However, 232 only few report directional interactions. One of these (17), reports cross-spectral 233 directionality between intracranial recordings in frontal cortex and the medial 234 temporal lobe in theta frequencies. One study on episodic memory suggests 235 directional information flow to and from hippocampus (28). Interestingly, our 236 analyses with intracranial recordings from posterior cortex revealed the most salient 237 findings in the beta frequencies, which is in line with hippocampal findings (15) and 238 theoretical considerations (29). For scalp EEG, the frequencies of synchronisation 239 found in the current study also included the theta-alpha frequencies, in line with 
240 scalp EEG findings during WM tasks $(4,6)$ and other tasks $(26)$ that activate

241 oscillations in long-range recurrent connections $(24,25)$.

242 In sum, these results corroborated earlier findings on the working memory network

243 and extended them by providing a physiological mechanism for the active replay of

244 memory items.

\section{METHODS}

$246 \quad$ Task

247 We used a modified Sternberg task in which the encoding of memory items and

248 their maintenance were temporally separated (Fig. 1a). Each trial started with a

249 fixation period $([-6,-5] \mathrm{s})$, followed by the stimulus $([-5,-3] \mathrm{s})$. The stimulus

250 consisted of a set of eight consonants at the center of the screen. The middle four,

251 six, or eight letters were the memory items, which determined the set size for the trial

252 (4, 6, or 8 respectively). The outer positions were filled with "X," which was never a

253 memory item. The participants read the letters and heard them spoken at the same

254 time. After the stimulus, the letters disappeared from the screen, and the

255 maintenance interval started ([-3, 0] s). A fixation square was shown throughout

256 fixation, encoding, and maintenance. After maintenance, a probe was presented.

257 The participants responded with a button press to indicate whether the probe was

258 part of the stimulus. The participants was instructed to respond as rapidly as

259 possible without making errors. After the response, the probe was turned off, and the

260 participants received acoustic feedback regarding whether the response was correct

261 or incorrect. The participants performed sessions of 50 trials in total, which lasted

262 approximately 10 min each. Trials with different set sizes were presented in a

263 random order, with the single exception that a trial with an incorrect response was

264 always followed by a trial with a set size of 4 . The task can be downloaded at

265 www.neurobs.com/ex files/expt view?id=266.

$266 \quad$ Participants

267 The participants in the study were patients with drug resistant focal epilepsy. To 268 investigate a potential surgical treatment of epilepsy, the patients were implanted

269 with intracranial electrodes. The participants provided written informed consent for

270 the study, which was approved by the institutional ethics review board (PB 2016- 
271 02055). The participants had normal or corrected-to-normal vision. For nine

272 participants $(4-13)$, the PSD and PLV has been reported in an earlier study (19).

273 Electrodes for sEEG, ECoG, and EEG

274 The depth electrodes (1.3 mm diameter, 8 contacts of $1.6 \mathrm{~mm}$ length, spacing

275 between contact centers 5 mm, ADTech®, Racine, WI, www.adtechmedical.com)

276 were stereotactically implanted into the hippocampus. Subdural grids and strips were

277 placed directly on the cortex according to the findings of the non-invasive presurgical

278 evaluations. Platinum electrodes with $4 \mathrm{~mm}^{2}$ contact surface and $1 \mathrm{~cm}$ inter-

279 electrode distances were used (ADTech®). In addition, scalp EEG electrodes were

280 placed at the sites of the 10-20 system with minor adaptations to avoid surgical scalp

281 lesions.

$282 \quad$ Electrode localization

283 To localize the ECoG grids and strips, we used the participants' postoperative $284 \mathrm{MR}$, aligned to CT and produced a 3D reconstruction of the participants' pial brain 285 surface. Grid/strip electrode coordinates were projected on the pial surface as 286 described in (30) (Fig. 2a, Fig. 3a,f).

287 The stereotactic sEEG electrodes were localized using post-implantation 288 computed tomography (CT) and post-implantation structural T1- weighted MRI 289 scans. The CT scan was registered to the post-implantation scan as implemented in 290 FieldTrip (31). A fused image of CT and MRI scans was produced and the electrode 291 contacts were marked visually. The contact positions were projected on a 292 parasagittal plane of MRI (Fig. 1b).

293 Recording setup, re-referencing, and preprocessing

294 All recordings were performed with Neuralynx ATLAS, sampling rate $4000 \mathrm{~Hz}$, 295 0.5-1000 Hz passband (Neuralynx, Bozeman MT, USA, www.neuralynx.com). ECoG 296 and sEEG were recorded against a common intracranial reference. ECoG was then 297 re-referenced against a sEEG contact in white matter. For each sEEG electrode, 298 hippocampal contacts were re-referenced against their closest sEEG contact in white 299 matter. The scalp EEG was recorded against an electrode near the vertex and was

300 then re-referenced to the averaged mastoid channels. All signals were downsampled 301 to $500 \mathrm{~Hz}$. Trials with large unitary artefacts in the scalp EEG were rejected. We 
302 focused on trials with high workload (set sizes 6 and 8) for further analysis. We used 303 the FieldTrip toolbox for data processing and analysis (32).

\section{Power spectral density}

305 We first calculated the relative power spectral density (PSD) in the time-frequency 306 domain (Fig. 2 b). Time-frequency maps for all trials were averaged. We used 3

307 multitapers with a window width of 10 cycles per frequency point, smoothed with 0.2 $308 \times$ frequency. We computed power in the frequency range 4-100 Hz with a time 309 resolution of $0.1 \mathrm{~s}$. The PSD during fixation $([-6.0,-5.0] \mathrm{s})$ served as a baseline for 310 the baseline correction (PSD(t) - PSD(fixation))/ PSD(fixation) for each time-

311 frequency point.

\section{$312 \quad$ Phase locking value}

313 To evaluate the functional connectivity of hippocampus and cortex, we calculated 314 the phase-locking value (PLV) between hippocampal sEEG channels and ECoG grid 315 (multitaper frequency transformation with 2 tapers based on Fourier transform, 316 frequency range $4-100 \mathrm{~Hz}$ with frequency resolution of $1 \mathrm{~Hz}$ ).

$$
P L V_{i, j}(f)=\frac{1}{N}\left|\sum_{n=1}^{N} \frac{X_{i}(f) \cdot\left(X_{j}(f)\right)^{*}}{\left|X_{i}(f)\right| \cdot\left|X_{j}(f)\right|}\right|
$$

317 where PLV $V_{i, j}$ is the PLV between channels $i, j, N$ is the number of trials, $X(f)$ is the 318 Fourier transform of $\mathrm{x}(\mathrm{t})$, and $(\cdot)^{*}$ represents the complex conjugate.

319 Using the spectra of the two-second epochs, phase differences were calculated 320 for each electrode pair (i,j) to quantify the inter-electrode phase coupling. The phase 321 difference between the two signals indexes the coherence between each electrode 322 pair and is expressed as the PLV. The PLV ranges between 0 and 1, with values 323 approaching 1 if the two signals show a constant phase relationship over all trials.

In our description of EEG frequency bands, we used theta $(4-8 \mathrm{~Hz})$, alpha (8-12 326 individual participants.

327 Spectral Granger causality

328 In order to evaluate the direction of information flow between the hippocampus 329 and the cortex, we calculated spectral non-parametric Granger causality (GC) as a 330 measure of directed functional connectivity. GC examines if the activity on one 331 channel can forecast activity in the target channel. In the spectral domain, GC 
332 measures the fraction of the total power that is contributed by the source to the 333 target. We transformed signals to the frequency domain using the multitaper method

334 (2 tapers, frequency range 4 to $100 \mathrm{~Hz}$ with frequency resolution of $0.5 \mathrm{~Hz}, \pm 2 \mathrm{~Hz}$ 335 smoothing) to reduce spectral leakage and control the frequency smoothing.

336 We used a non-parametric spectral approach to measure the interaction in the 337 channel pairs at a given interval time (33). In this approach, the spectral transfer 338 matrix is obtained from the Fourier transform of the data. We used the FieldTrip 339 toolbox to factorize the transfer function $\mathrm{H}(\mathrm{f})$ and the noise covariance matrix $\Sigma$. The 340 transfer function and the noise covariance matrix was then employed to calculate the 341 total and the intrinsic power, $\mathrm{S}(\mathrm{f})=\mathrm{H}(\mathrm{f}) \Sigma \mathrm{H}^{*}(\mathrm{f})$, through which we calculated the

342 Granger interaction in terms of power fractions contributed from the source to the 343 target.

$$
G C_{Y \rightarrow X} \rightarrow=\ln \frac{S_{x x(f)}}{\tilde{S}_{x x(f)}}
$$

345 where $S_{x x(f)}$ is the total power and $\tilde{S}_{x x(f)}$ the instantaneous power. To improve 346 legibility, we present GC as Granger \% = GC*100 (Fig. 2 d,e).

347 To illustrate the time course of GC over time, we calculated time-frequency maps 348 for the selected pairs with scalp channels and averaged these maps over 349 participants.

\section{$350 \quad$ Statistics}

351 To analyze statistical significance, we used cluster-based nonparametric

352 permutation tests. To assess the significance of PLV and GC, we compared the true 353 values to a null distribution. We recomputed GC after shuffling the trial number for a 354 single channel in the pair, while keeping the trial number of the other channel 355 constant. We repeated this $\mathrm{n}=200$ times to create a null distribution of GC. The null 356 distribution was exploited to calculate the percentile threshold $P=0.05$. We mark the 357 frequency range of significant GC with a blue bar for encoding (dark blue spectrum 358 exceeds light blue spectrum, information flow from cortex to hippocampus) and with 359 a red bar for maintenance (dark red spectrum exceeds light red spectrum, 360 information flow from hippocampus to cortex).

361 For comparisons between two groups, we used the paired cluster based 362 permutation test. We created a null distribution by performing $N=200$ random 363 permutations. The significance threshold was established at $P=0.05$. 


\section{Acknowledgments}

365 We thank the physicians and the staff at Schweizerische Epilepsie-Klinik for their 366 assistance and the patients for their participation. We acknowledge grants awarded

367 by the Swiss National Science Foundation (SNSF 320030_176222 to J. S.) and

368 SNSF Ambizione fellowship (PZ00P3_167836 to P. M.). The funders had no role in 369 the design or analysis of the study.

370 Author contributions: J.S. designed the experiments. J.S. performed the 371 experiments. V.D. and J.S. analyzed the data. L.S. performed surgery. P.M.

372 visualized the grid electrode position, V.D. and J.S. wrote the manuscript. All of the 373 authors reviewed the final version of the manuscript.

374 Competing interests: All authors declare that they have no competing interests.

375 Ethical considerations: The participant provided written informed consent for the 376 study, which was approved by the institutional ethics review board (PB 2016-02055).

377 Data availability: All data needed to evaluate the conclusions in the paper are 378 present in the paper. The task can be downloaded at 379 www.neurobs.com/ex files/expt view?id=266. The raw data will be made publicly 380 available after acceptance of the manuscript.

\section{$381 \quad$ FIGURE CAPTIONS}

$382 \quad$ Figure 1. Task and recording sites.

383 a) In the task, sets of consonants are presented and have to be memorized. The set 384 size $(4,6$ or 8 letters) determines WM workload. In each trial, presentation of a 385 letter string (encoding period, $2 \mathrm{~s}$ ) is followed by a delay (maintenance period, 3 386 s). After the delay, a probe letter is presented. Participants indicate whether the 387 probe was in the letter string or not.

388 b) Response accuracy decreases with set size (71 sessions).

389 c) Reaction time increases with set size (53 ms/item).

390 d) The tip locations of the hippocampal sEEG electrodes for all participants $(N=15)$ 391 are projected on the parasagittal plane $x=25.2 \mathrm{~mm}$. 
392

393

394

395

396

397

398

399

400

401

402

403

404

405

406

407

408

409

410

411

412

413

414

415

416

417

418

419

420

421

422

423

424

Figure 2. Encoding and replay of letters in Participant 1

a) Location of the ECoG contacts over temporal and parietal cortex for Participant 1. Contacts record from auditory cortex (C2, cyan) and occipital-parietal cortex (H3, magenta).

b) The relative power spectral density (PSD) in the parietal contact (magenta) shows gamma (>40 Hz) during encoding [-5 -3] s while the subject sees and hears the letters. Sustained beta activity $(11-14 \mathrm{~Hz})$ appears towards the end of the maintenance period [-3 0] s.

c) Hippocampal PSD shows sustained beta activity towards the end of maintenance.

d) Phase locking value (PLV) between cortex and hippocampal sEEG in beta during maintenance. PLV (16-29 Hz) is maximal in contact C2 over auditory cortex.

e) Spectral Granger causality. During encoding, auditory cortex (contact C2) predicts hippocampus (8-18 Hz, dark blue curve exceeds light blue curve). During maintenance, hippocampal sEEG predicts auditory cortex (8-14 Hz, dark red curve exceeds light red curve).

f) The Granger time-frequency map illustrates the spectra of panel e. During encoding, net information ( $\Delta$ Granger) starts to flow from auditory cortex to hippocampus (blue). During maintenance the information flow is reversed from hippocampus to auditory cortex (red) indicating the replay of letters in memory.

\section{Figure 3. Encoding and replay of letters in two participants with ECoG}

a) Location of the ECoG contacts in Participant 2. The most anterior strip contact (cyan) records from auditory cortex.

b) The relative power spectral density (PSD) in the temporal scalp EEG electrode (T3) shows beta $(14-25 \mathrm{~Hz})$ during encoding [-5 -3] s while the subject sees and hears the letters. Sustained theta activity $(6-9 \mathrm{~Hz})$ appears towards the end of the maintenance period [-3 0] s.

c) Hippocampal PSD shows sustained alpha-beta activity $(9-21 \mathrm{~Hz})$ towards the end of maintenance.

d) Spectral Granger causality. During encoding, the auditory cortex predicts hippocampus (9-16 Hz, dark blue curve exceeds light blue curve). During maintenance, hippocampal sEEG predicts auditory cortex $(9-16 \mathrm{~Hz}$, dark red curve exceeds light red curve). 
425 e) The time-frequency map illustrates the time course of $\Delta$ Granger in Participant 3.

426 f) Location of the ECoG contacts in Participant 3. The most posterior contact

427 (cyan) records from visual cortex.

$428 \mathrm{~g})$ The relative power spectral density (PSD) in the blue contact shows gamma

429 during encoding while the subject sees the letters. Sustained alpha activity (7-12

$430 \mathrm{~Hz}$ ) appears towards the end of the maintenance period.

431 h) Hippocampal PSD shows sustained beta activity $(13-22 \mathrm{~Hz})$ towards the end of 432 maintenance.

433 i) Spectral Granger causality. During encoding, the occipital ECoG (cyan contact)

434 predicts hippocampus (10-18 Hz, dark blue curve exceeds light blue curve).

435 During maintenance, hippocampal sEEG predicts ECoG $(9-18 \mathrm{~Hz}$, dark red

$436 \quad$ curve exceeds light red curve).

437 j) The time-frequency map illustrates the time course of $\Delta$ Granger in Participant 3.

438 Bars: frequency range of significant $\Delta$ Granger $(p<0.05)$, cluster-based

439 nonparametric permutation test against a null distribution with scrambled trials during 440 encoding (dark blue) and maintenance (dark red), respectively.

441 Figure 4. Granger causality between hippocampal sEEG and scalp EEG

442 Granger causality spectra for hippocampal sEEG and scalp EEG for the electrode

443 pair with maximal Granger causality for all participants $(\mathrm{N}=15)$. During encoding, the

444 net Granger ( $\triangle$ Granger) indicates information flow from scalp EEG to hippocampal

445 sEEG (blue bar). During maintenance, $\triangle$ Granger indicates information flow from

446 hippocampal sEEG to scalp EEG to (red bar). Each panel carries the name of the

447 scalp EEG electrode site.

448 Bars: frequency range of significant $\Delta$ Granger $(\mathrm{p}<0.05)$, cluster-based nonparametric

449 permutation test against a null distribution with scrambled trials during encoding

450 (dark blue) and maintenance (dark red), respectively. Colors of Granger spectra

451 indicating information flow:

452 dark blue, EEG to hippocampus during encoding;

453 light blue, hippocampus to EEG during encoding;

454 dark red, hippocampus to EEG during maintenance;

455 light red, EEG to hippocampus during maintenance. 

behavior.

458 a) The time-frequency map of net Granger causality ( $\Delta$ Granger), averaged over all $459 \mathrm{~N}=15$ participants, illustrates the time course of the information flow from scalp 460 EEG to hippocampus (blue) during encoding and from hippocampus to scalp EEG 461 (red) during maintenance.

462 b) For correct trials, the maximal $\Delta$ Granger is negative during encoding (blue, cortex

$463 \rightarrow$ hippocampus) and positive during maintenance (red, hippocampus $\rightarrow$ cortex)

464 for all participants (triangles: pairs with scalp EEG, circles: pairs with ECoG, $P=$

$465 \quad 0.005$, paired cluster based permutation test).

466 c) For incorrect trials, the maximal $\Delta$ Granger is highly variable $(P=0.0647$, paired

467 cluster based permutation test, not significant).

468 d) Bidirectional information flow between posterior cortical sites and hippocampus in

469 the working memory network. The data suggest a surprisingly simple model of

470 information flow during the task. During encoding, letter strings are verbalized as a

471 melody. The incoming information flows from sensory cortex to hippocampus.

472 During maintenance, participants actively recall and rehearse the melody. The

473 Granger causality indicates the information flow from hippocampus to cortex as

474 the physiological basis for the replay of the memory items.

475

Figure S1. Further analyses in Participant 1

477 a) The relative power spectral density (PSD) in the temporal contact (grid contact $478 \quad \mathrm{C} 2$, cyan in Figure 2 a) shows gamma $(>40 \mathrm{~Hz})$ during encoding [-5 -3] s while the 479 subject sees and hears the letters, thus confirming the anatomical location of this 480 contact over auditory cortex.

481 b) Phase locking value (PLV) between hippocampus and grid contact C2. To 482 assess the significance of the difference of the PLV between different task 483 periods, we used the same permutation test as for the evaluation of significance 484 for Granger causality. We mark the frequency range of significant PLV $(18-30 \mathrm{~Hz})$ 485 with a red bar when the PLV spectrum during maintenance (red spectrum) 486 exceeds the PLV spectrum during fixation (black spectrum).

487 c) Spectral Granger causality during encoding. In the $8-18 \mathrm{~Hz}$ range, auditory 488 cortex (contact C2) is maximal in predicting hippocampus. 
489

490

491

492

493

494

495

496

497

498

499

500

501

502

503

504

505

506

507

508

509

510

511

512

513

514

515

516

517

518

519

520

521

522

523

524

525

526

527

528

529

530

531

532

533

d) Spectral Granger causality during maintenance. In the 8-14 $\mathrm{Hz}$ range, hippocampus predicts auditory cortex (contact C2) and parietal cortex (contact F7). Axis labels denote contact names.

1. Baddeley A (2003) Working memory: looking back and looking forward. Nat Rev Neurosci 4(10):829-839.

2. Christophel TB, Klink PC, Spitzer B, Roelfsema PR, \& Haynes J-D (2017) The Distributed Nature of Working Memory. Trends in Cognitive Sciences 21(2):111-124.

3. Michels L, Moazami-Goudarzi M, Jeanmonod D, \& Sarnthein J (2008) EEG alpha distinguishes between cuneal and precuneal activation in working memory. Neurolmage 40(3):1296-1310.

4. Sarnthein J, Petsche H, Rappelsberger P, Shaw GL, \& von Stein A (1998) Synchronization between prefrontal and posterior association cortex during human working memory. Proc Natl Acad Sci U S A 95(12):7092-7096.

5. Tuladhar AM, et al. (2007) Parieto-occipital sources account for the increase in alpha activity with working memory load. Hum Brain Mapp 28(8):785-792.

6. Polania R, Nitsche MA, Korman C, Batsikadze G, \& Paulus W (2012) The importance of timing in segregated theta phase-coupling for cognitive performance. Curr Biol 22(14):1314-1318.

7. Näpflin M, Wildi M, \& Sarnthein J (2008) Test-retest reliability of EEG spectra during a working memory task. Neurolmage 43(4):687-693.

8. Bidelman GM, Brown JA, \& Bashivan P (2021) Auditory cortex supports verbal working memory capacity. Neuroreport 32(2):163-168.

9. Pavlov YG \& Kotchoubey B (2020) Oscillatory brain activity and maintenance of verbal and visual working memory: A systematic review. Psychophysiology:e13735.

10. Cogan GB, et al. (2017) Manipulating stored phonological input during verbal working memory. Nat Neurosci 20(2):279-286.

11. Raghavachari S, et al. (2001) Gating of human theta oscillations by a working memory task. J Neurosci 21(9):3175-3183.

12. Rizzuto DS, et al. (2003) Reset of human neocortical oscillations during a working memory task. Proceedings of the National Academy of Sciences 100(13):7931-7936.

13. Maris E, van Vugt M, \& Kahana M (2011) Spatially distributed patterns of oscillatory coupling between high-frequency amplitudes and low-frequency phases in human iEEG. Neurolmage 54(2):836-850.

14. van Vugt MK, Schulze-Bonhage A, Litt B, Brandt A, \& Kahana MJ (2010) Hippocampal gamma oscillations increase with memory load. J Neurosci 30(7):2694-2699.

15. Leszczyński M, Fell J, \& Axmacher N (2015) Rhythmic Working Memory Activation in the Human Hippocampus. Cell Rep 13(6):1272-1282.

16. Schwiedrzik CM, et al. (2018) Medial prefrontal cortex supports perceptual memory. Current Biology 28(18):R1094-R1095. 
534 17. Johnson EL, et al. (2018) Dynamic frontotemporal systems process space

535

536

537

538

539

540

541

542

543

544

545

546

547

548

549

550

551

552

553

554

555

556

557

558

559

560

561

562

563

564

565

566

567

568

569

570

571

572

573

574

575

576

577

578 and time in working memory. PLOS Biology 16(3):e2004274.

18. Johnson EL, et al. (2019) Spectral Imprints of Working Memory for Everyday Associations in the Frontoparietal Network. Front Syst Neurosci 12:65.

19. Boran E, et al. (2019) Persistent hippocampal neural firing and hippocampalcortical coupling predict verbal working memory load. Sci Adv 5(3):eaav3687.

20. Kamiński J, et al. (2017) Persistently active neurons in human medial frontal and medial temporal lobe support working memory. Nat Neurosci 20(4):590601.

21. Kornblith S, Quian Quiroga R, Koch C, Fried I, \& Mormann F (2017) Persistent Single-Neuron Activity during Working Memory in the Human Medial Temporal Lobe. Curr Biol 27(7):1026-1032.

22. Rutishauser U, Reddy L, Mormann F, \& Sarnthein J (2021) The Architecture of Human Memory: Insights from Human Single-Neuron Recordings. The Journal of Neuroscience 41(5):883-890.

23. Hsieh LT \& Ranganath C (2014) Frontal midline theta oscillations during working memory maintenance and episodic encoding and retrieval. Neurolmage 85 Pt 2:721-729.

24. Fries $P(2015)$ Rhythms for Cognition: Communication through Coherence. Neuron 88(1):220-235.

25. Pesaran B, et al. (2018) Investigating large-scale brain dynamics using field potential recordings: analysis and interpretation. Nat Neurosci 21(7):903-919.

26. Solomon EA, et al. (2017) Widespread theta synchrony and high-frequency desynchronization underlies enhanced cognition. Nat Commun 8(1):1704.

27. Boran E, et al. (2020) Dataset of human medial temporal lobe neurons, scalp and intracranial EEG during a verbal working memory task. Sci Data 7(1):30.

28. Griffiths BJ, et al. (2019) Directional coupling of slow and fast hippocampal gamma with neocortical alpha/beta oscillations in human episodic memory. Proc Natl Acad Sci U S A 116(43):21834-21842.

29. Gelastopoulos A, Whittington MA, \& Kopell NJ (2019) Parietal low beta rhythm provides a dynamical substrate for a working memory buffer. Proc Natl Acad Sci U S A 116(33):16613-16620.

30. Groppe DM, et al. (2017) iELVis: An open source MATLAB toolbox for localizing and visualizing human intracranial electrode data. $J$ Neurosci Methods 281:40-48.

31. Stolk A, et al. (2017) Integrated analysis of anatomical and electrophysiological human intracranial data. bioRxiv.

32. Oostenveld R, Fries P, Maris E, \& Schoffelen J-M (2011) FieldTrip: Open Source Software for Advanced Analysis of MEG, EEG, and Invasive Electrophysiological Data. Computational Intelligence and Neuroscience 2011:9.

33. Bastos AM \& Schoffelen J-M (2016) A Tutorial Review of Functional Connectivity Analysis Methods and Their Interpretational Pitfalls. Frontiers in systems neuroscience 9:175-175. 
a
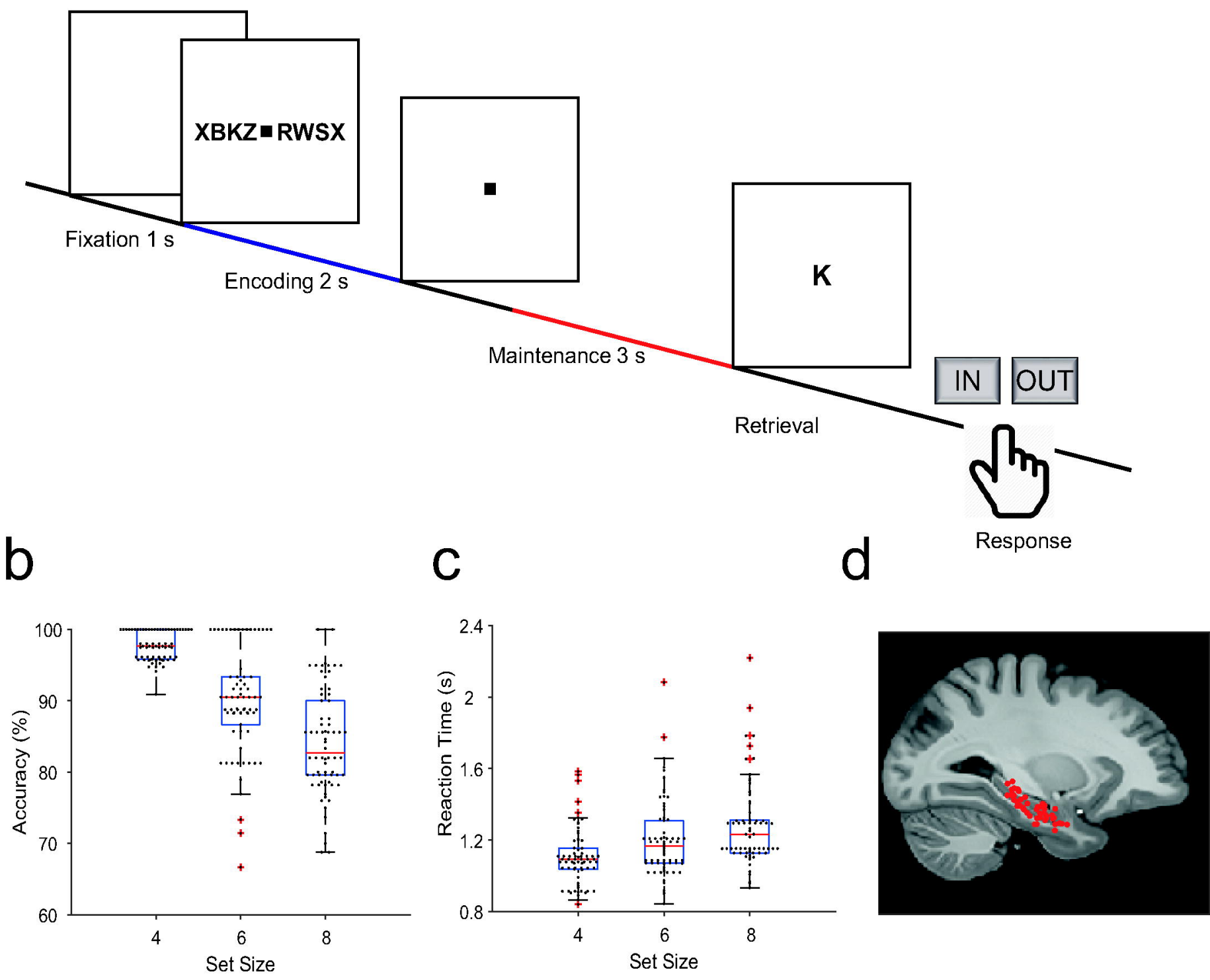
a

d

8
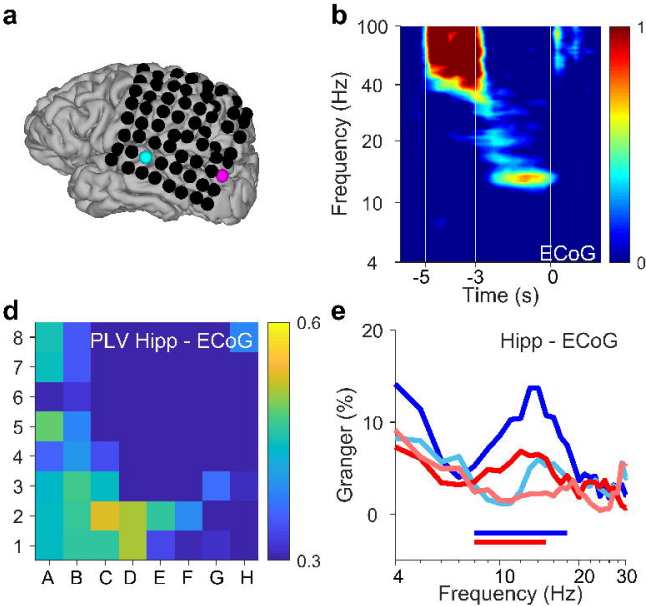

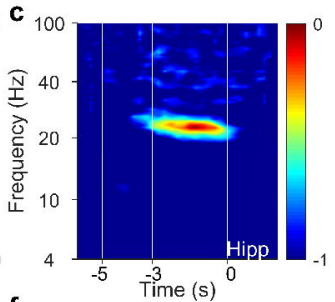

f

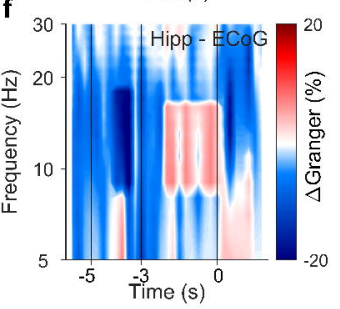


a

\section{ECOG}

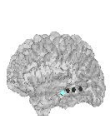

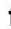

f b c

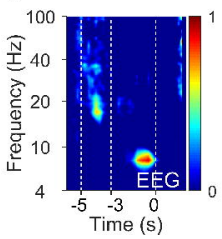

g

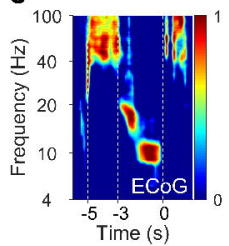

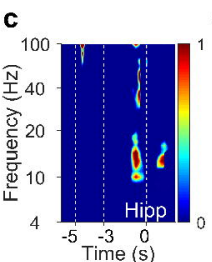

d e

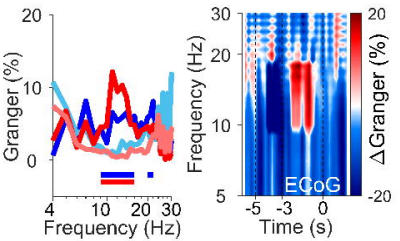

h
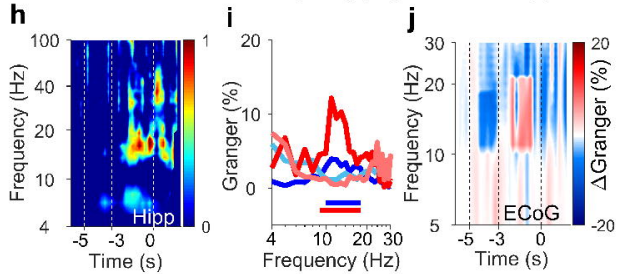


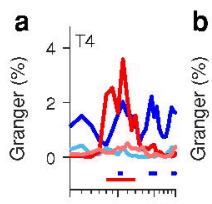

$4 \quad 10 \quad 2030$

Frequency $(\mathrm{Hz})$

f
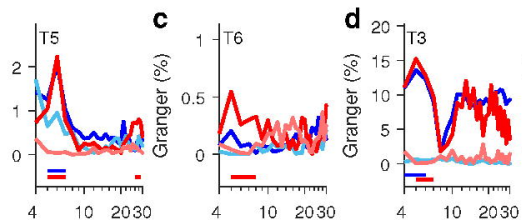

Frequency $(\mathrm{Hz})$

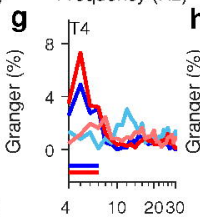

$4 \quad 10 \quad 2030$

Frequency $(\mathrm{Hz})$

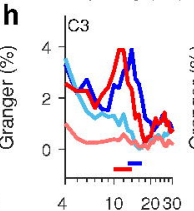

$4 \quad 10 \quad 2030$

Frequency $(\mathrm{Hz})$

i Frequency $(\mathrm{Hz})$

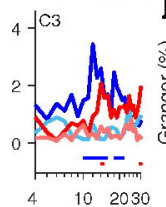

Frequency $(\mathrm{Hz})$

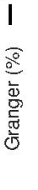

Frequency $(\mathrm{Hz})$

Frequency $(\mathrm{Hz})$
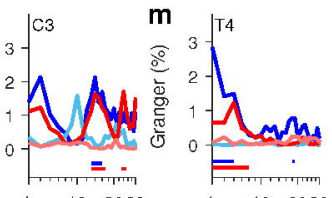

Frequency $(\mathrm{Hz})$

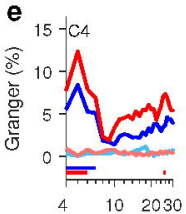

Frequency $(\mathrm{Hz})$

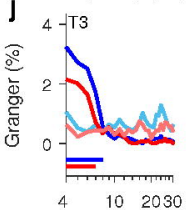

Frequency $(\mathrm{Hz})$

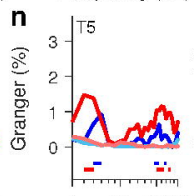

$4 \quad 10 \quad 2030$

Frequency $(\mathrm{Hz})$

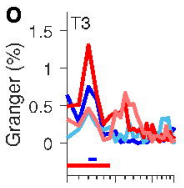

$4 \quad 10 \quad 2030$

Frequency $(\mathrm{Hz})$ 
